\title{
Graphene as gain medium for broadband lasers
}

\author{
Roland Jago, ${ }^{1}$ Torben Winzer, ${ }^{2}$ Andreas Knorr, ${ }^{2}$ and Ermin Malic ${ }^{1, *}$ \\ ${ }^{1}$ Department of Applied Physics, Chalmers University of Technology, 41296 Gothenburg, Sweden \\ ${ }^{2}$ Institute of Theoretical Physics, Technical University Berlin, Hardenbergstrasse 36, Berlin 10623, Germany
}

(Received 14 November 2014; revised manuscript received 13 July 2015; published 6 August 2015)

\begin{abstract}
Efficient nonradiative carrier recombination strongly counteracts the appearance of optical gain in graphene. Based on a microscopic and fully quantum-mechanical study of the coupled carrier, phonon, and photon dynamics in graphene, we present a strategy to obtain a long-lived gain: Integrating graphene into a high quality photonic crystal nanocavity and applying a high-dielectric substrate suppresses the nonradiative recombination channels and gives rise to pronounced coherent light emission. This suggests the design of graphene-based laser devices covering a broad spectral range.
\end{abstract}

DOI: 10.1103/PhysRevB.92.085407

\section{INTRODUCTION}

Visible and infrared fiber lasers are the basis for information technology, while microwave and radio-frequency emitters build the backbone of wireless communications. Due to the lack of efficient sources of terahertz light, there is a technological gap between these two frequency ranges [1]. The ongoing search for novel gain materials has brought ultrathin layered nanomaterials into the focus of current research. Graphene, a single layer of carbon atoms, exhibits a linear and gapless band structure around the Dirac point $[2,3]$. This unique electronic dispersion offers a broad spectrum of optically active states including the terahertz region [4]. This extraordinary feature has already been technologically exploited in graphene-based photodetectors covering a wide range of frequencies [5-9] as well as graphene-based saturable absorbers converting the continuous-wave output of lasers into a train of ultrashort optical pulses [10-12]. Recently, hot photoluminescence has been observed across the entire visible spectrum even exceeding the energy of the excitation reflecting radiative recombination of excited carriers [13-17]. In the strong excitation regime, a spectrally broad population inversion (PI) has been measured [18,19] and theoretically predicted [20-22]. The excited carriers become quickly redistributed filling the optically active states in the vicinity of the Dirac point, where the reduced density of states gives rise to a relaxation bottleneck and a buildup of a PI resulting in optical gain. However, nonradiative carrier recombination channels reduce the accumulation of charge carriers accounting for a decay of the population inversion on a femtosecond time scale making it unsuitable for technological applications [21].

In this work, we address the question whether a long-lived optical gain can be achieved in graphene. This presents the key prerequisite for the realization of graphene-based broadband laser devices that could also operate in the technologically relevant terahertz spectral region. To answer this question, we perform a microscopic and fully quantum-mechanical investigation of the coupled carrier, phonon, and photon dynamics in strongly pumped graphene within a cavity. We shed light on the occurring carrier-carrier, carrier-phonon, and carrier-photon interactions on the same microscopic footing allowing us to track the way of nonequilibrium carriers resolved in time and

\footnotetext{
*ermin.malic@chalmers.se
}

PACS number(s): 78.67.Wj, 73.22.Pr, 78.45.+h

energy. The performed microscopic treatment of Coulombinduced and phonon-assisted relaxation processes as well as radiative and nonradiative carrier recombination channels unravels the driving microscopic mechanism underlying the emission of light from graphene: Figure 1 shows a sketch of the competing relaxation processes for optically pumped carriers. Based on our calculations, we propose a strategy of how to achieve long-lived gain resulting in coherent laser light emission from graphene: (i) enhancement of the carrierlight interaction by integrating graphene into a high quality photonic crystal nanocavity $[23,24]$ and at the same time (ii) reduction of the efficiency of Coulomb-induced nonradiative recombination by considering graphene on a highdielectric substrate. To address the quantum statistics of the emitted light and to answer the question of whether coherent laser light can be achieved, we determine the temporal evolution of the second-order autocorrelation function.

\section{THEORETICAL APPROACH}

The study is performed within the formalism of the density matrix theory [25-30] offering microscopic access to temporally and spectrally resolved carrier, phonon, and photon dynamics [31-34]. The many-particle Hamilton operator $H=H_{0}+H_{\mathrm{c}, \mathrm{c}}+H_{\mathrm{c}, \mathrm{p}}+H_{\mathrm{c}, \mathrm{pt}}$ consists of the (i) freecarrier, phonon, and photon contribution $H_{0}$, (ii) carriercarrier $H_{\mathrm{c}, \mathrm{c}}$ and (iii) carrier-phonon interaction $H_{\mathrm{c}, \mathrm{p}}$ accounting for Coulomb- and phonon-induced intraband scattering and nonradiative recombination processes, respectively, and finally (iv) carrier-photon interaction $H_{\mathrm{c}, \mathrm{pt}}$ describing the radiative recombination of excited carriers; cf. Fig. 1.

Treating all many-particle interactions on the same quantum-mechanical level, we derive graphene luminescence equations:

$$
\begin{gathered}
\dot{\rho}_{\mathbf{k}}^{\lambda}(t)=\Gamma_{\mathbf{k} \lambda}^{\mathrm{in}}(t)\left[1-\rho_{\mathbf{k}}^{\lambda}(t)\right]-\Gamma_{\mathbf{k} \lambda}^{\mathrm{out}}(t) \rho_{\mathbf{k}}^{\lambda}(t) \pm 2 \sum_{\gamma}\left|\tilde{M}_{\gamma \mathbf{k}}^{v c}\right|^{2} \\
\times \operatorname{Re}\left[{ }^{1} S_{\gamma \mathbf{k}}^{v c}(t)\right] \pm P(t)\left[\rho_{\mathbf{k}}^{v}(t)-\rho_{\mathbf{k}}^{c}(t)\right] \\
\dot{n}_{\mathbf{q}}^{j}(t)=\Gamma_{\mathbf{q} j}^{e m}(t)\left[n_{\mathbf{q}}^{j}(t)+1\right]-\Gamma_{\mathbf{q} j}^{\mathrm{abs}}(t) n_{\mathbf{q}}^{j}(t) \\
\quad-\gamma_{p h}^{j}\left[n_{\mathbf{q}}^{j}(t)-n_{\mathbf{q}, B}^{j}\right] \\
\dot{n}_{p t}^{\gamma}(t)=2 \sum_{\mathbf{k}}\left|\tilde{M}_{\gamma \mathbf{k}}^{v c}\right|^{2} \operatorname{Re}\left[{ }^{1} S_{\gamma \mathbf{k}}^{v c}(t)\right]-2 \kappa_{\gamma}\left[n_{p t}^{\gamma}(t)-n_{B}^{\gamma}\right]
\end{gathered}
$$




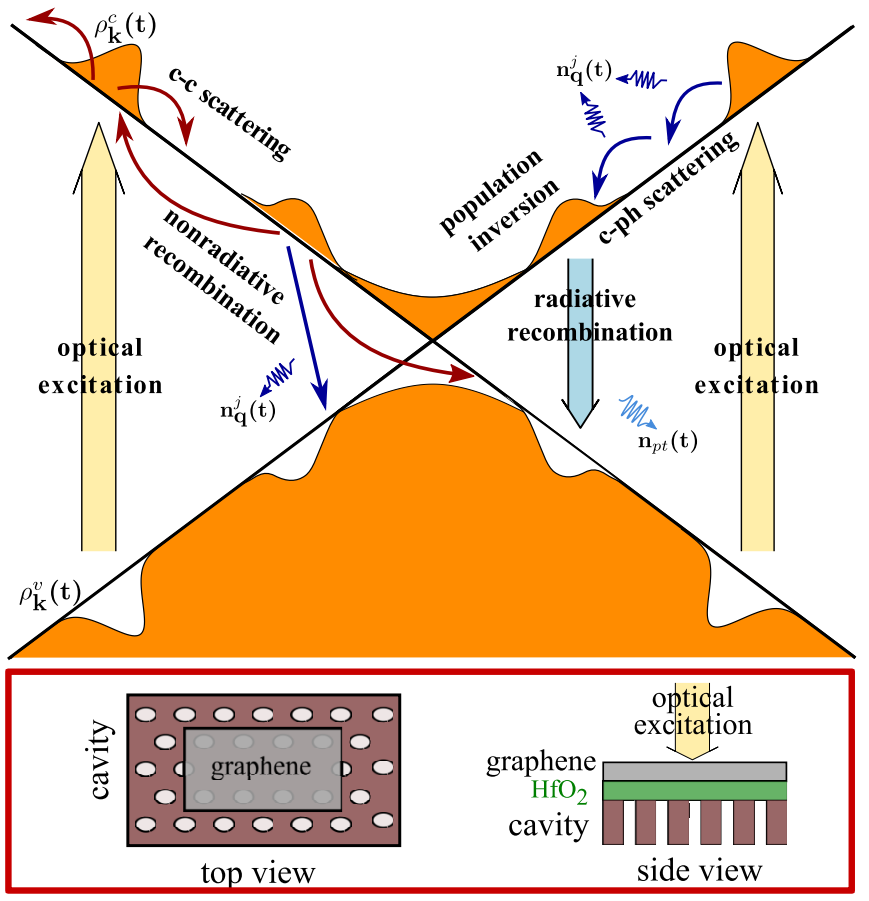

FIG. 1. (Color online) Schematic illustration of the carrier, phonon, and photon dynamics in optically pumped graphene. Nonequilibrium carriers relax towards lower energies via carriercarrier (c-c) and carrier-phonon (c-ph) scattering. Above a threshold pump strength, a population inversion occurs giving rise to an increased efficiency for radiative recombination. However, at the same time, the accumulation of carriers also enhances the competing nonradiative recombination channels which reduce the achieved population inversion. The schematic pictures at the bottom illustrate the top and the side view of graphene integrated within a planar photonic crystal cavity.

This is a coupled set of differential equations for the carrier occupation probability $\rho_{\mathbf{k}}^{\lambda}(t)=\left\langle a_{\mathbf{k} \lambda}^{+} a_{\mathbf{k} \lambda}\right\rangle$ in the valence $(\lambda=$ $v)$ and the conduction band $(\lambda=c)$ with the electronic momentum $\mathbf{k}$, the phonon number $n_{\mathbf{q}}^{j}(t)=\left\langle b_{\mathbf{q} j}^{+} b_{\mathbf{q} j}\right\rangle$ for different optical and acoustic phonon modes $j$ with the phonon momentum $\mathbf{q}$, and finally the photon number $n_{p t}^{\gamma}(t)=\left\langle c_{\gamma}^{+} c_{\gamma}\right\rangle$ in the mode $\gamma$. Here, we have introduced $a_{\mathbf{k} \lambda}^{+}$and $a_{\mathbf{k} \lambda}$ as creation and annihilation operators for carriers, $b_{\mathbf{q} j}^{+}$and $b_{\mathbf{q} j}$ for phonons, and $c_{\gamma}^{+}$and $c_{\gamma}$ for photons, respectively.

A nonequilibrium carrier distribution is achieved by optically exciting graphene with a symmetric steady-state-like pulse

$$
P(t)=P_{0} \exp \left[-\left(E-E_{0}\right)^{2} / 2 \sigma_{E}^{2}\right] \exp \left[-\left(t-t_{0}\right)^{6} / 2 \sigma_{t}^{6}\right]
$$

characterized by the pumping rate $P_{0}=0.05 \mathrm{fs}^{-1}$, the pump energy $E_{0}=1.5 \mathrm{eV}$, and spectral and temporal widths $0.2 \mathrm{eV}$ and $20 \mathrm{ps}$, respectively. The characteristics of the excitation pulse correspond to typical values that can be easily realized by standard pulsed lasers. The time- and momentum-dependent in- and out-scattering rates $\Gamma_{\mathbf{k} \lambda}^{\text {in/out }}(t)$ appearing in Eq. (1) include carrier-carrier and carrier-phonon scattering channels (for more details, see Refs. [31,32]). The carrier-photon interaction leads to additional terms driven by the onephoton-assisted polarization ${ }^{1} S_{\gamma \mathbf{k}}^{v c}(t)=\left\langle a_{\mathbf{k} v}^{+} a_{\mathbf{k} c} c_{\gamma}^{+}\right\rangle^{c}(t)$. The dynamics of electrons in the conduction and the valence band is symmetric [ + in Eq. (1) stands for $\lambda=v$ and - for $\lambda=c$ ]. The dynamics of the phonon number $n_{\mathbf{q}}^{j}$ is driven by the emission and absorption rates [31,32] $\Gamma_{\mathbf{q} j}^{\mathrm{em} / \mathrm{abs}}(t)$; cf. Eq. (2). The temporal evolution of the emitted photons $n_{p t}^{\gamma}$ is given in Eq. (3) focusing on the dominant photon mode $\gamma_{0}$ determined by the cavity. Equation (1) also contains the contribution of the spontaneous emission into nonlasing modes, i.e., all terms with $\gamma \neq \gamma_{0}$ in the appearing sum. The phonon and photon lifetimes are denoted by $\gamma_{p h}$ and $\kappa_{\gamma}$. The latter is related to the quality factor $Q$ of the cavity via $\kappa_{\gamma_{0}}=\frac{\omega_{\gamma_{0}}}{2 Q}$ with the eigenfrequency $\omega_{\gamma_{0}}$. Furthermore, $n_{\mathbf{q}, B}^{j}$ and $n_{B}^{\gamma}$ are the initial Bose distributions for the phonon and photon numbers.

Here, we focus on the quantum optical aspect of the theory and discuss in more detail the Hamilton operator $H_{\mathrm{c}, \mathrm{pt}}$ describing the carrier-photon interaction in a cavity,

$$
H_{\mathrm{c}, \mathrm{pt}}=i \hbar \sum_{\mathbf{k}} \tilde{M}_{\gamma_{0} \mathbf{k}}^{v c}\left(a_{\mathbf{k} v}^{+}, a_{\mathbf{k} c} c_{\gamma_{0}}^{+}-a_{\mathbf{k} c}^{+} a_{\mathbf{k} v} c_{\gamma_{0}}\right),
$$

with the carrier-photon matrix element $\tilde{M}_{\gamma_{0} \mathbf{k}}^{v c}=$ $\frac{e_{0}}{m_{0}} \sqrt{\frac{\hbar}{2 \omega_{\gamma_{0}} \varepsilon_{0} V}} \hat{\mathbf{e}}_{\gamma_{0}} \cdot \mathbf{M}_{\mathbf{k}}^{v c}$. determining the strength of the interaction. It depends on the properties of the optical cavity characterized by a dominant mode $\gamma_{0}$ with the eigenfrequency $\omega_{0}$ and the cavity volume $V$. In particular, the carrier-photon interaction is the strongest for small cavity frequencies and small cavity volumina. Furthermore, the coupling has the same symmetry as the semiclassical optical matrix element [32] $\mathbf{M}_{\mathbf{k}}^{v c}=\langle\mathbf{k} v|\nabla| \mathbf{k} c\rangle$ and depends on the polarization $\hat{\mathbf{e}}_{\gamma}$.

The emitted light intensity from optically pumped graphene is characterized by the photon dynamics $\dot{n}_{p t}^{\gamma}(t)$ driven by the one-photon-assisted polarization ${ }^{1} S_{\gamma \mathbf{k}}^{v c}$ that describes the recombination of excited carriers between the conduction and the valence band in the state $\mathbf{k}$ accompanied by the emission of a photon in the mode $\gamma$. Assuming that no coherent excitation are allowed, i.e., $\left\langle c_{\gamma}^{+}\right\rangle=\left\langle c_{\gamma}\right\rangle=0$, and factorizing quantities with four electronic operators, we obtain

$$
\begin{aligned}
{ }^{1} \dot{S}_{\gamma \mathbf{k}}^{v c}(t)= & i \Delta \omega_{\gamma \mathbf{k}}^{v c}(t){ }^{1} S_{\gamma \mathbf{k}}^{v c}(t)+\left[1-\rho_{\mathbf{k}}^{v}(t)\right] \rho_{\mathbf{k}}^{c}(t) \\
& -\left[\rho_{\mathbf{k}}^{v}(t)-\rho_{\mathbf{k}}^{c}(t)\right] n_{p t}^{\gamma}(t)-{ }^{2} S_{\gamma \mathbf{k}}^{v v}(t)+{ }^{2} S_{\gamma \mathbf{k}}^{c c}(t),
\end{aligned}
$$

with $\Delta \omega_{\gamma \mathbf{k}}^{v c}=\omega_{\mathbf{k} v}-\omega_{\mathbf{k} c}+\omega_{\gamma}+i\left[\kappa_{\gamma}+\gamma_{\mathbf{k}}(t)\right]$. The equation includes the free-particle oscillation and the damping of ${ }^{1} S_{\gamma \mathbf{k}}^{v c}$ as well as the processes of spontaneous and stimulated emission and absorption of light. The time- and momentumdependent damping $\gamma_{\mathbf{k}}(t)$ is determined by Coulomb- and phonon-induced scattering processes, which have been explicitly included into the calculations. The appearing two-photon assisted densities ${ }^{2} S_{\gamma \mathbf{k}}^{\lambda \lambda}=\left\langle a_{\mathbf{k} \lambda}^{+} a_{\mathbf{k} \lambda} c_{\gamma}^{+} c_{\gamma}\right\rangle^{c}(t)$ contain corrections stemming from higher-order many-particle processes. The contribution of the spontaneous emission to the carrier occupation can be separately solved within the Markov approximation resulting in [29] $\left.\dot{\rho}_{\mathbf{k}}^{\lambda}(t)\right|_{\mathrm{sp}}= \pm \frac{1-\beta_{\mathbf{k}}}{\tau_{\mathbf{k}}^{s p}}\left[1-\rho_{\mathbf{k}}^{v}(t)\right] \rho_{\mathbf{k}}^{c}(t)$ with the spontaneous emission factor $\beta_{\mathbf{k}}$ that is defined as the ratio of spontaneous emission into the lasing mode $\gamma_{0}$ to the total spontaneous emission into all possible photon modes $\gamma$. Here, $\tau_{\mathbf{k}}^{\text {sp }}$ denotes the total spontaneous emission rate. At the considered cavity energy of $400 \mathrm{meV}$, we obtain the values 
$\beta_{\gamma_{0}}=0.1$ and $\tau_{\gamma_{0}}^{\mathrm{sp}}=500 \mathrm{ps}$. The $+(-)$ sign stands for the occupation in the valence (conduction) band.

To characterize the emitted light, we investigate the photon statistics, which is given by the second-order autocorrelation function [35]:

$$
g_{\gamma}^{(2)}(t)=\frac{\left\langle c_{\gamma}^{+} c_{\gamma}^{+} c_{\gamma} c_{\gamma}\right\rangle(t)}{\left\langle c_{\gamma}^{+} c_{\gamma}\right\rangle^{2}(t)}=2+\frac{n_{p t, 2}^{\gamma}(t)}{\left[n_{p t}^{\gamma}(t)\right]^{2}} .
$$

Since quantities including four photonic operators $n_{p t, 2}^{\gamma}(t)=$ $\left\langle c_{\gamma}^{+} c_{\gamma}^{+} c_{\gamma} c_{\gamma}\right\rangle^{c}(t)$ appear, we have to treat the dynamics of photons up to the fourth order in the Born approximation. The equation of motion for $n_{p t, 2}^{\gamma}(t)$ reads

$$
\dot{n}_{p t, 2}^{\gamma}(t)=4 \sum_{\mathbf{k}}\left|\tilde{M}_{\gamma \mathbf{k}}^{v c}\right|^{2} \operatorname{Re}\left[{ }^{3} S_{\gamma \mathbf{k}}^{v c}(t)\right]-4 \kappa_{\gamma} n_{p t, 2}^{\gamma}(t) .
$$

The temporal evolution of the three-photon assisted polarization ${ }^{3} S_{\gamma \mathbf{k}}^{v c}(t)=\left\langle a_{\mathbf{k} v}^{+} a_{\mathbf{k} c} c_{\gamma}^{+} c_{\gamma}^{+} c_{\gamma}\right\rangle^{c}(t)$ is given by

$$
\begin{aligned}
{ }^{3} \dot{S}_{\gamma \mathbf{k}}^{v c}(t)= & i\left\{\omega_{\mathbf{k} v}-\omega_{\mathbf{k} c}+\omega_{\gamma}+i\left[3 \kappa_{\gamma}+\gamma_{\mathbf{k}}(t)\right]\right\}{ }^{3} S_{\gamma \mathbf{k}}^{v c}(t) \\
& -2\left|\tilde{M}_{\gamma \mathbf{k}}^{v c}\right|^{2}\left[{ }^{1} S_{\gamma \mathbf{k}}^{v c}(t)\right]^{2}-\left[\rho_{\mathbf{k}}^{v}(t)-\rho_{\mathbf{k}}^{c}(t)\right] n_{p t, 2}^{\gamma}(t) \\
& +2\left[1-\rho_{\mathbf{k}}^{v}(t)\right]^{2} S_{\gamma \mathbf{k}}^{c c}(t)-2 \rho_{\mathbf{k}}^{c}(t){ }^{2} S_{\gamma \mathbf{k}}^{v v}(t) .
\end{aligned}
$$

Note that the two-photon-assisted densities ${ }^{2} S_{\gamma \mathbf{k}}^{\lambda \lambda}(t)$ occur in the equations for both photon-assisted polarizations ${ }^{1} S_{\gamma \mathbf{k}}^{v c}(t)$ and ${ }^{3} S_{\gamma \mathbf{k}}^{v c}(t)$, such that they link the photon number to the photon statistics. Their equation of motion reads

$$
\begin{aligned}
{ }^{2} \dot{S}_{\gamma \mathbf{k}}^{\lambda \lambda}(t)= & -\left[2 \kappa_{\gamma}+\gamma_{\mathbf{k}}(t)\right]{ }^{2} S_{\gamma \mathbf{k}}^{\lambda \lambda}(t) \pm 2\left|\tilde{M}_{\gamma \mathbf{k}}^{v c}\right|^{2} \\
& \times \operatorname{Re}\left\{{ }^{3} S_{\gamma \mathbf{k}}^{v c}(t)+\left[n_{p t}^{\gamma}(t)+1-\rho_{\mathbf{k}}^{\lambda}(t)\right]{ }^{1} S_{\gamma \mathbf{k}}^{v c}(t)\right\}
\end{aligned}
$$

Now, we have a closed set of graphene luminescence equations [Eqs. (1)-(9)] allowing us to address the temporally and spectrally resolved dynamics and the quantum statistics of emitted photons from optically pumped graphene within a cavity. In particular, having access to the second-order autocorrelation function $g_{\gamma}^{(2)}$, we are able to distinguish coherent laser light characterized by the Poisson statistics [35] with $g_{\gamma}^{(2)}=1$ from thermal light $\left(g_{\gamma}^{(2)}>1\right)$ and nonclassical light $\left(g_{\gamma}^{(2)}<1\right)$.

\section{CARRIER, PHONON, AND PHOTON DYNAMICS}

Solving the graphene luminescence equations, we have full microscopic access to time- and energy resolved carrier, phonon, and photon dynamics in graphene. We assume the graphene layer to be integrated in a high quality photonic crystal nanocavity, as already successfully realized in previous studies $[23,24]$. Such cavities are known to have the potential to reach extremely high quality factors $Q$ and ultrasmall cavity volumes $V$. For this study, we assume $Q=22500$ and $V \approx 10^{-13} \mathrm{~cm}^{3}$ according to the work of Akahane and co-workers [23]. We investigate the emission of photons in the cavity mode of $E_{0}=400 \mathrm{meV}$ (corresponding to the carrier energy of $200 \mathrm{meV}$ ), where the maximal population inversion (PI) is reached. While the volume of the cavity can be rather large, its size in the direction perpendicular to the graphene layer has a direct impact on the number of emitted photons and
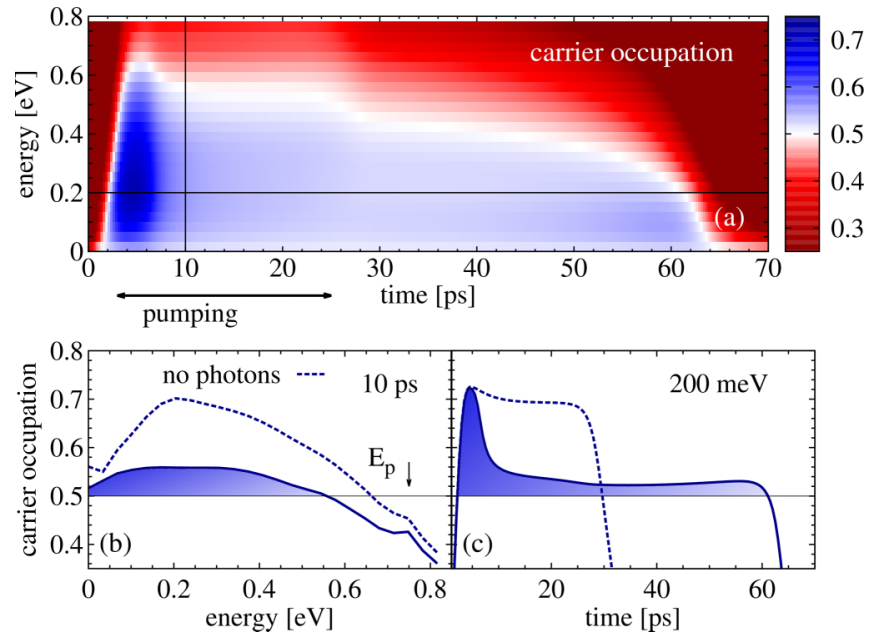

FIG. 2. (Color online) Temporally and spectrally resolved carrier dynamics. (a) The surface plot shows the nonequilibrium dynamics of optically pumped carriers in the conduction band. Blue area displays the region of population inversion with $\rho(E, t)>0.5$. The carrier occupation is shown (b) as a function of the carrier energy for the fixed time of $10 \mathrm{ps}$ after the switch-on of the optical excitation pulse and (c) as a function of time at the fixed carrier energy of $200 \mathrm{meV}$. The width of the pump pulse is $20 \mathrm{ps}$ and the pumping energy $E_{p}$ is $1.5 \mathrm{eV}$ corresponding to a carrier energy of $0.75 \mathrm{eV}$. The dashed lines show the carrier dynamics without photons illustrating the crucial impact of the carrier-photon coupling.

has to be in the range of few $100 \mathrm{~nm}$ to obtain the emission of coherent laser light. This can be realized even for terahertz frequencies by integrating graphene into planar photonic crystal nanocavities $[23,24]$. The dependence on the quality factor will be addressed in the discussion part of this paper.

To partially suppress nonradiative recombination processes, we assume that graphene lies on a high-dielectric substrate. Here, we have exemplary considered hafnium dioxide $\left(\mathrm{HfO}_{2}\right)$ with a static dielectric constant [36] of $\varepsilon=25$. The substrate is assumed to induce an electrostatic background potential that screens the Coulomb interaction within the graphene layer. In this situation, the static limit of the dielectric function is a well justified approximation [26].

In the following, we first discuss the time- and energyresolved dynamics of nonequilibrium carriers and phonons after optical pumping. Having established a long-lived population inversion, we then investigate the dynamics of photons and of photon-photon correlations determining the quantum statistics of emitted light.

Carrier dynamics. The temporal and spectral evolution of optically pumped carriers in graphene within a photonic crystal nanocavity is illustrated in Fig. 2. We observe a pronounced spectrally broad PI, i.e., the occupation of carriers in the conduction band $\rho(E, t)$ is higher than 0.5 [blue region in Fig. 2(a)]. Our calculations reveal that the nonequilibrium carriers are quickly scattered to energetically lower states via carrier-carrier and carrier-phonon interactions leading to an ultrafast accumulation of carriers. A spectrally broad PI up to energies slightly below the pump energy $E_{p}$ is obtained; cf. Fig. 2(b). To study the dynamics of the microscopic mechanism behind the PI in more detail, we show in Fig. 2(c) 


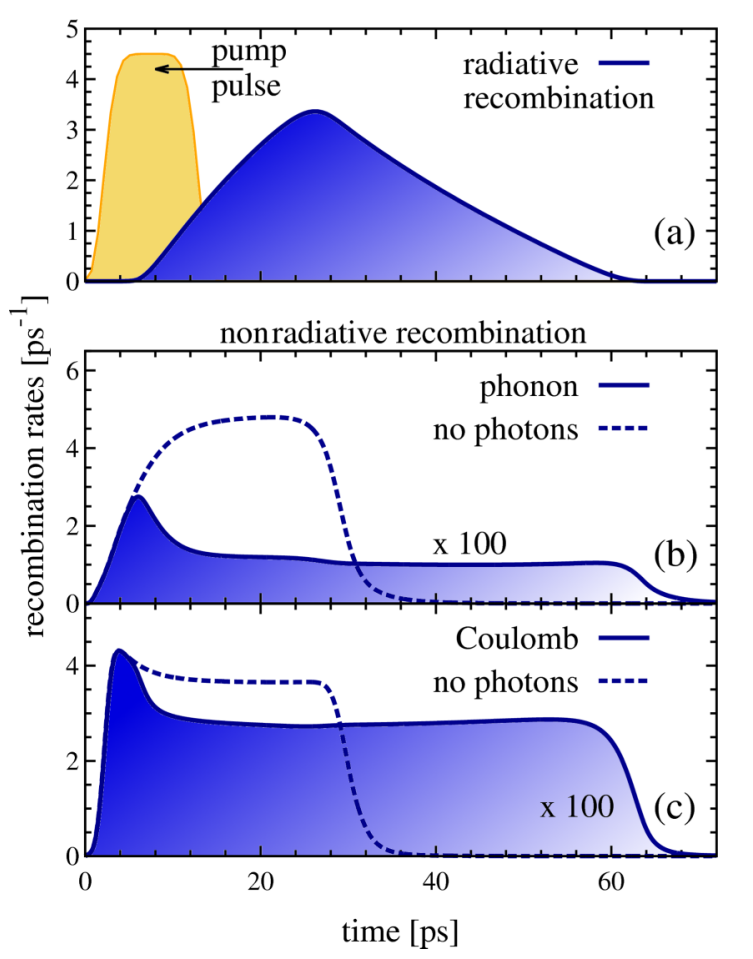

FIG. 3. (Color online) Radiative and nonradiative recombination rates. Temporal evolution of (a) radiative, (b) phonon-assisted, and (c) Coulomb-induced recombination rates. The corresponding scattering channels are in direct competition with each other during the entire dynamics. Note that the nonradiative recombination rates are two orders of magnitude smaller than the radiative process due to the enhancement of the carrier-light coupling within a high quality nanocavity and due to the suppression of Coulomb interaction through substrate-induced screening. The dashed lines show the rates without taking into account the carrier-photon coupling illustrating the crucial impact of photons on the efficiency of nonradiative recombination channels during and after the optical excitation.

the carrier occupation as a function of time at the fixed carrier energy of $200 \mathrm{meV}$ with and without the carrier-photon coupling. In the full calculation including the impact of photons (solid line), the PI lives on a time scale of $60 \mathrm{ps}$. We find pronounced carrier occupation values of up to 0.7 as long as the pump pulse reaches its maximum. Then, the occupation quickly decreases predominantly due to the efficient radiative recombination. The loss of carriers via radiative and nonradiative recombination processes can be partially compensated during and after the pumping by intraband scattering from energetically higher states; cf. Fig. 1. The interplay of radiative emission and absorption processes on the one side and nonradiative recombination channels and Coulomb- and phononinduced intraband scattering processes on the other side results in a quasiequilibrium keeping the carrier occupation at a stationary value slightly above 0.5 on a long time scale. Once the loss of carriers cannot be compensated anymore, the carrier occupation quickly drops to small values determined by the thermal distribution. Applying stronger and longer excitation pulses, we can further increase the PI lifetime.

The crucial role of radiative processes is demonstrated in calculations neglecting the dynamics of photons [dashed line in Fig. 2(c)]. Here, we find a decay of the PI on a time scale

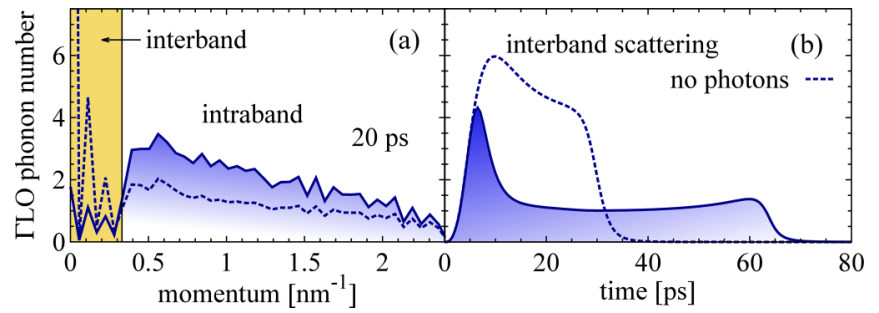

FIG. 4. (Color online) Temporally and spectrally resolved phonon dynamics. (a) Spectral resolution of the exemplary $\Gamma L O$ phonons at a fixed time of $20 \mathrm{ps}$ after the switch-on of the optical excitation. The yellow-shaded region emphasizes phonons with the momentum $q<0.3 \mathrm{~nm}^{-1}$ inducing interband scattering of excited carriers (nonradiative recombination), whereas phonons with $q>$ $0.3 \mathrm{~nm}^{-1}$ contribute to intraband scattering [32]. The dashed lines show the dynamics without photons revealing more pronounced recombination on the one hand and considerably suppressed intraband processes on the other hand. The results are numerically converged despite the zigzag shape of the data that can be ascribed to the numerical inaccuracy of vector addition in polar coordinate grids. (b) Temporal resolution of the $\Gamma L O$ phonon dynamics at a fixed momentum of $0.18 \mathrm{~nm}^{-1}$ describing the efficiency of nonradiative carrier recombination induced by the emission of $\Gamma L O$ phonons.

of the pump pulse ( $20 \mathrm{ps}$ ). In the absence of light emission, nonradiative recombination channels are predominant giving rise to a quick decay of the PI. This behavior is well reflected by the temporal evolution of the corresponding radiative and nonradiative recombination rates, which are illustrated in Fig. 3. A necessary condition for a long-lived PI is a considerable suppression of the efficient processes of Auger recombination [37-44]. These Coulomb-induced interband processes bringing excited electrons down to the valence band are known to be by orders of magnitude higher than radiative recombination in free-suspended graphene [21]. Therefore, in this work, we introduce a high-dielectric substrate, which considerably reduces the efficiency of Coulomb-induced nonradiative processes. At the same time, implementing graphene into a photonic crystal nanocavity, we strongly enhance the carrier-light interaction. With this recipe, we achieve radiative recombination rates that are two orders of magnitude higher than the competing nonradiative processes; cf. Fig. 3.

Phonon dynamics. The emission of light is in direct competition to phonon-assisted interband scattering. It depopulates the states close to the Dirac point, which in turn drives the phonon-assisted intraband scattering to fill these states; cf. Fig. 1. We find a considerable nonequilibrium phonon distribution over a broad momentum range reflecting significant carrier-phonon scattering. Our calculations reveal that indeed the interband scattering is significantly suppressed, while at the same time the efficiency of intraband processes is enhanced in the presence of photons; cf. Fig. 4. Besides the discussed Coulomb- and phonon-induced dynamics, impurity scattering can contribute to the carrier relaxation. However, this elastic-scattering process accounts only for orientational relaxation [45], i.e., it does not counteract a long-lived population inversion.

Photon dynamics. Having understood the carrier and phonon dynamics, we now focus on the temporal evolution 


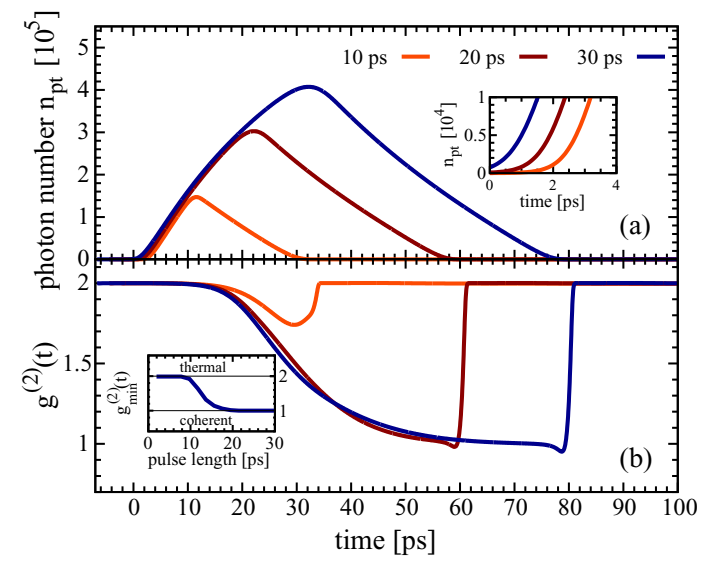

FIG. 5. (Color online) Photon dynamics and statistics. Illustration of the temporal evolution of (a) photons emitted from optically pumped graphene within a photonic crystal nanocavity and of (b) the second-order autocorrelation function $g^{(2)}(t)$ expressing the statistics of emitted light for different widths of the excitation pulse (at a constant pump rate $P_{0}$ ). The cavity energy is fixed to $400 \mathrm{meV}$. The inset in (a) corresponds to a zoom-in revealing the initial turn-on dynamics. The inset in (b) shows the minimum of $g_{\min }^{(2)}$ as a function of the pulse width revealing that coherent laser light [characterized by $g^{(2)}(t)=1$ ] occurs for excitation of $20 \mathrm{ps}$ and longer.

of emitted photons from optically pumped graphene within a nanocavity. Figure 5(a) shows $n_{p t}(t)$ at the fixed cavity energy of $400 \mathrm{meV}$ for different pump pulse widths modeling the behavior towards stationary pumping. In the first few picoseconds, the number of emitted photons increases slowly and can be ascribed to the spontaneous emission; cf. also the inset of Fig. 5(a). After this characteristic delay time that is determined by the pump strength, the process of induced emission boosts the number of photons as long as the pump pulse is switched on. The pumping maintains the carrier supply that is necessary to keep the PI on a high level. After pumping, the number of photons gradually decreases directly reflecting the behavior of the radiative recombination rate; cf. Fig. 3(a). As soon as the carrier loss cannot be compensated anymore by Coulomb- and phonon-induced in-scattering of further carriers from energetically higher states, the number of photons quickly drops, since the PI vanishes and the stimulated emission does not contribute anymore. Figure 5(a) reveals that the decay of the number of emitted photons can be controlled by the length of the optical excitation. The longer the pump pulse, the more stable is the PI resulting in a more pronounced emission of light.

Photon statistics. To investigate whether the emitted light corresponds to coherent laser light, we calculate the photon statistics that is determined by the second-order autocorrelation function $g^{(2)}$. Perfectly coherent laser light follows the Poissonian photon statistics characterized by $g^{(2)}=1$ describing random time intervals between the emitted photons [35]. Figure 5(b) shows the temporal evolution of $g^{(2)}$ for different pump widths. As long as the photon dynamics is dominated by the processes of spontaneous emission, we find $g^{(2)} \approx 2$ corresponding to the value expected for thermal chaotic light. After few picoseconds, $g^{(2)}$ starts to decrease reaching the value of 1 after few tens of ps, i.e., optically excited graphene within a nanocavity indeed emits coherent
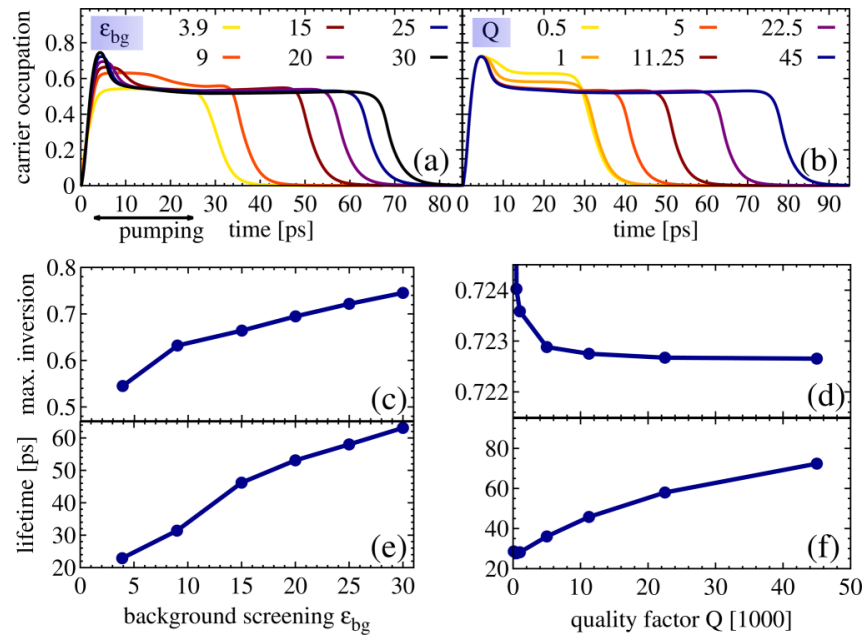

FIG. 6. (Color online) Impact of the substrate-induced screening and the cavity quality factor. Temporal evolution of the carrier occupation for (a) different dielectric background constants $\varepsilon_{b g}$ (at a constant cavity quality factor of 22500) and (b) for different quality factors $Q$ (at a constant $\varepsilon_{b g}=25$ ). (c),(d) Maximal population inversion and (e),(f) its lifetime are shown as a function of $\varepsilon_{b g}$ and $Q$, respectively. We find that the PI lifetime and its maximum clearly increase for substrates with a higher dielectric background constant. In contrast, for enhanced quality factors the maximal value of the PI is slightly reduced, while the PI lifetime strongly increases.

laser light. Whether perfectly coherent light can be obtained depends on the duration of the pumping. For pump pulse widths in the range of $20 \mathrm{ps}, g^{(2)}=1$ can be reached; cf. the inset of Fig. 5(b).

\section{DISCUSSION}

The main message of our paper is that a long-lived gain and pronounced coherent laser light emission can be achieved by integrating graphene into a photonic crystal nanocavity and by applying a high-dielectric substrate. The crucial parameters here are (i) the dielectric background constant $\varepsilon_{b g}$ determining the screening of the Coulomb interaction through the presence of the substrate and (ii) the quality factor $Q$ of the photonic crystal nanocavity. Here, we discuss the carrier dynamics for a series of different substrates and cavity quality factors, in particular focusing on their impact on the PI lifetime.

Figure 6(a) illustrates the carrier dynamics for different substrates characterized by the static dielectric background function $\varepsilon_{b g}$. We find that for low-dielectric substrates, such as $\mathrm{SiO}_{2}$ with $\varepsilon_{b g}=3.9$, the maximal PI is very small and its lifetime only lasts during the excitation pulse. The larger $\varepsilon_{b g}$, the higher is the PI and the longer is its lifetime; cf. Figs. 6(c) and 6(e). The explanation is that the quasiequilibrium between the radiative emission and absorption processes on the one side and nonradiative recombination channels and Coulomb- and phonon-induced intraband scattering processes on the other side (cf. the schematic Fig. 1) last longer when the Coulomb interaction is strongly suppressed by efficient substrate-induced screening. For free-standing graphene or for graphene on low-dielectric substrates, the Coulomb-induced 

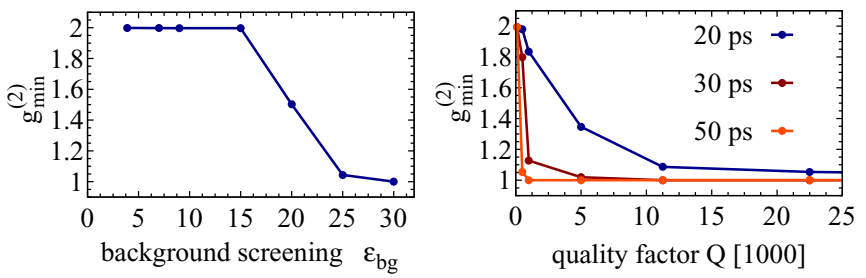

FIG. 7. (Color online) Photon statistics in dependence of the substrate and the cavity. The minimum of the second-order autocorrelation function $g_{\min }^{(2)}$ in dependence of (a) the dielectric background constants $\varepsilon_{b g}$ and (b) the cavity quality factor $Q$. We find that $\varepsilon_{b g}>15$ and $Q>500$ (depending on the excitation length) are required to obtain emission of nonthermal light $\left(g_{\min }^{(2)}<2\right)$ from graphene.

recombination is the dominant process strongly limiting the population inversion and its lifetime.

A similar behavior is observed when the cavity quality factor is varied; cf. Fig. 6(b). Here, the main observation is that the value of the maximal population inversion only slightly changes with increasing $Q$ [Fig. 6(d)], whereas the PI lifetime becomes strongly enhanced [Fig. 6(f)] reaching values of approximately $80 \mathrm{ps}$ for high quality photonic crystal cavities. Increasing the carrier-photon interaction within such a cavity enhances the efficiency of both radiative emission and absorption processes. As a result, the accumulation of carriers close to the Dirac point does not significantly change. However, high quality factors $Q$ give rise to a long photon lifetime $\kappa_{\gamma}^{-1}$, since $Q$ and $\kappa_{\gamma}$ are inverse to each other. Consequently, in high quality cavities, the excited energy remains longer in the carrier-photon system resulting in larger PI lifetimes.

Finally, Fig. 7 shows the dependence of the minimum of the second-order autocorrelation function $g^{(2)}$ as a function of $\varepsilon_{b g}$ and $Q$. We find that one needs substrates with $\varepsilon_{b g}>15$ to achieve a long-lived population inversion resulting in the emission of nonthermal light that is characterized by $g^{(2)}<2$; cf. Fig. 7(a). The required value for the quality factor of the cavity strongly depends on the length of the optical excitation. While for pulse widths of $20 \mathrm{fs}, Q$ values in the range of 10000 are needed, for an excitation width of 50 fs already a quality factor of approximately 1000 is sufficient to obtain coherent laser light characterized by $g^{(2)}=1$; cf. Fig. 7(b).

\section{CONCLUSIONS}

Based on a microscopic and fully quantum-mechanic study we have gained fundamental insights into the coupled carrier, phonon, and photon dynamics in optically pumped graphene within a photonic crystal nanocavity. We present a strategy to achieve coherent laser light emission from graphene by using a high quality photonic crystal cavity and a high-dielectric substrate. The obtained microscopic insights shed light on elementary many-particle processes behind the amplification of light in graphene presenting a crucial step towards the realization of future graphene-based nanolasers operating at a broad range of frequencies.

\section{ACKNOWLEDGMENTS}

We acknowledge financial support from the German Science Foundation (DFG) through the priority program SPP 1459 (E.M. and T.W.) and the Research Training Group GRK 1558 (A.K. and R.J.). Furthermore, E.M. acknowledges financial support from the Swedish Research Council (VR) and the EU Graphene Flagship (Contract No. CNECT-ICT-604391).
[1] R. Köhler, A. Tredicucci, F. Beltram, H. E. Beere, E. H. Linfield, A. G. Davies, D. A. Ritchie, R. C. Iotti, and I. F. Rossi, Nature (London) 417, 156 (2002).

[2] K. S. Novoselov, A. K. Geim, S. V. Morozov, D. Jiang, Y. Zhang, S. V. Dubonos, I. V. Grigorieva, and A. A. Firsov, Science 306, 666 (2004).

[3] A. K. Geim and K. S. Novoselov, Nat. Mater. 6, 183 (2007).

[4] P. Tassin, T. Koschny, and C. M. Soukoulis, Science 341, 620 (2013).

[5] F. Xia, T. Mueller, Y.-m. Lin, A. Valdes-Garcia, and P. Avouris, Nat. Nanotechnol. 4, 839 (2009).

[6] T. Mueller, F. Xia and P. Avouris, Nat. Photon. 4, 297 (2010).

[7] T. Echtermeyer, L. Britnell, P. Jasnos, A. Lombardo, R. Gorbachev, A. Grigorenko, A. Geim, and A. F. K. Novoselov, Nat. Commun. 82, 458 (2011).

[8] M. Furchi, A. Urich, A. Pospischil, G. Lilley, K. Unterrainer, H. Detz, P. Klang, A. M. Andrews, W. Schrenk, G. Strasser, and T. Mueller, Nano Lett. 12, 2773 (2012).

[9] M. Engel, M. Steiner, A. Lombardo, A. C. Ferrari, H. v. Löhneysen, P. Avouris, and R. Krupke, Nat. Commun. 3, 906 (2012).
[10] Z. Sun, T. Hasan, F. Torrisi, D. Popa, G. Privitera, F. Wang, F. Bonaccorso, D. M. Basko, and A. C. Ferrari, ACS Nano 4, 803 (2010).

[11] F. Bonaccorso, Z. Sun, T. Hasan, and A. C. Ferrari, Nat. Photon. 4, 611 (2010).

[12] P. Avouris and C. Dimitrakopoulos, Mater. Today 15, 86 (2012).

[13] C. H. Lui, K. F. Mak, J. Shan, and T. F. Heinz, Phys. Rev. Lett. 105, 127404 (2010).

[14] W.-T. Liu, S. W. Wu, P. J. Schuck, M. Salmeron, Y. R. Shen, and F. Wang, Phys. Rev. B 82, 081408(R) (2010).

[15] R. J. Stöhr, R. Kolesov, J. Pflaum, and J. Wrachtrup, Phys. Rev. B 82, 121408 (2010).

[16] A. Säynätjoki, L. Karvonen, J. Riikonen, W. Kim, S. Mehravar, R. A. Norwood, N. Peyghambarian, H. Lipsanen, and K. Kieu, ACS Nano 7, 8441 (2013).

[17] T. Winzer, R. Ciesielski, M. Handloser, A. Comin, A. Hartschuh, and E. Malic, Nano Lett. 15, 1141 (2015).

[18] T. Li, L. Luo, M. Hupalo, J. Zhang, M. C. Tringides, J. Schmalian, and J. Wang, Phys. Rev. Lett. 108, 167401 (2012).

[19] I. Gierz, J. C. Petersen, M. Mitrano, C. Cacho, I. C. E. Turcu, E. Springate, A. Stöhr, A. Köhler, U. Starke, and A. Cavalleri, Nat. Mater. 12, 1119 (2013). 
[20] V. Ryzhii, M. Ryzhii, and T. Otsuji, J. Appl. Phys. 101, 083114 (2007).

[21] T. Winzer, E. Malić, and A. Knorr, Phys. Rev. B 87, 165413 (2013).

[22] F. Wendler and E. Malic, Sci. Rep. 5, 12646 (2015).

[23] Y. Akahane, T. Asano, B. S. Song, and S. Noda, Nature (London) 425, 944 (2003).

[24] X. Gan, K. F. Mak, Y. Gao, Y. You, F. Hatami, J. Hone, T. F. Heinz, and D. Englund, Nano Lett. 12, 5626 (2012).

[25] M. Kira, F. Jahnke, W. Hoyer, and S. Koch, Prog. Quantum Electron. 23, 189 (1999).

[26] H. Haug and S. W. Koch, Quantum Theory of the Optical and Electronic Properties of Semiconductors (World Scientific, Singapore, 2004).

[27] M. Kira and S. W. Koch, Semiconductor Quantum Optics (Cambridge University Press, Cambridge, England, 2012).

[28] F. Rossi and T. Kuhn, Rev. Mod. Phys. 74, 895 (2002).

[29] C. Gies, J. Wiersig, M. Lorke, and F. Jahnke, Phys. Rev. A 75, 013803 (2007).

[30] A. Knorr, S. Hughes, T. Stroucken, and S. W. Koch, Chem. Phys. 210, 27 (1996).

[31] E. Malic and A. Knorr, Graphene and Carbon Nanotubes: Ultrafast Optics and Relaxation Dynamics (Wiley-VCH, New York, 2013).

[32] E. Malic, T. Winzer, E. Bobkin, and A. Knorr, Phys. Rev. B 84, 205406 (2011).
[33] E. Malic, T. Winzer, and A. Knorr, Appl. Phys. Lett. 101, 213110 (2012).

[34] T. Winzer, A. Knorr, M. Mittendorff, S. Winnerl, M.-B. Lien, D. Sun, T. B. Norris, M. Helm, and E. Malic, Appl. Phys. Lett. 101, 221115 (2012).

[35] M. O. Scully and M. S. Zubairy, Quantum Optics, 1st ed. (Cambridge University Press, Cambridge, England, 1997).

[36] G. D. Wilk, R. M. Wallace, and J. M. Anthony, J. Appl. Phys. 89, 5243 (2001).

[37] T. Winzer, A. Knorr, and E. Malic, Nano Lett. 10, 4839 (2010).

[38] T. Winzer and E. Malić, Phys. Rev. B 85, 241404 (2012).

[39] L. Pirro, A. Girdhar, Y. Leblebici, and J.-P. Leburton, J. Appl. Phys. 112, 093707 (2012).

[40] D. Brida, A. Tomadin, C. Manzoni, Y. J. Kim, A. Lombardo, S. Milana, R. R. Nair, K. S. Novoselov, A. C. Ferrari, G. Cerullo, and M. Polini, Nat. Commun. 4, 1987 (2013).

[41] T. Plötzing, T. Winzer, E. Malic, D. Neumaier, A. Knorr, and H. Kurz, Nano Lett. 14, 5371 (2014).

[42] I. Gierz, F. Calegari, S. Aeschlimann, M. C. Cervantes, C. Cacho, R. T. Chapman, E. Springate, S. Link, U. Starke, C. R. Ast, and A. Cavalleri, arXiv:1506.00120.

[43] F. Wendler, A. Knorr, and E. Malic, Nat. Commun. 5, 3703 (2014).

[44] M. Mittendorff, F. Wendler, E. Malic, A. Knorr, M. Orlita, M. Potemski, C. Berger, W. A. de Heer, H. Schneider, M. Helm, and S. Winnerl, Nat. Phys. 11, 75 (2015).

[45] B. Y. Sun and M. W. Wu, New J. Phys. 15, 083038 (2013). 